Revue

Revue de l'histoire des religions

del'histoire

des religions

$1 \mid 2014$

Varia

François Dolbeau, Prophètes, apôtres et disciples dans les traditions chrétiennes d'Occident. Vies brèves et listes en latin

Bruxelles, Société des Bollandistes (« Subsidia hagiographica », 92), 2012

Cécile Lanéry

(2) OpenEdition

Journals

Édition électronique

URL : http://journals.openedition.org/rhr/8217

DOI : $10.4000 /$ rhr.8217

ISSN : 2105-2573

Éditeur

Armand Colin

Édition imprimée

Date de publication : 1 mars 2014

Pagination : 140-143

ISBN : 978-2200929107

ISSN : 0035-1423

Référence électronique

Cécile Lanéry, "François Dolbeau, Prophètes, apôtres et disciples dans les traditions chrétiennes

d'Occident. Vies brèves et listes en latin », Revue de l'histoire des religions [En ligne], 1 | 2014, mis en ligne le 13 mai 2014, consulté le 22 septembre 2020. URL : http://journals.openedition.org/rhr/8217 ; DOI : https://doi.org/10.4000/rhr.8217

Ce document a été généré automatiquement le 22 septembre 2020

Tous droits réservés 


\section{François Dolbeau, Prophètes, apôtres et disciples dans les traditions chrétiennes d'Occident. Vies brèves et listes en latin}

Bruxelles, Société des Bollandistes (« Subsidia hagiographica », 92), 2012

\section{Cécile Lanéry}

\section{RÉFÉRENCE}

François Dolbeau, Prophètes, apôtres et disciples dans les traditions chrétiennes d'Occident.

Vies brèves et listes en latin, Bruxelles, Société des Bollandistes ( Subsidia

hagiographica », 92), 2012, 24,7 cm, 437 p., $79 €$, ISBN 978-2-87365-027-8.

1 En 2005, François Dolbeau avait publié, sous le titre Sanctorum societas, un ample recueil d'articles consacrés à l'hagiographie; il poursuit aujourd'hui dans la même collection, avec ce volume dédié aux prophètes, aux apôtres et aux disciples du Christ, un domaine qu'il a grandement contribué à défricher côté latin. Depuis 1986, date de son premier article sur le sujet, il n'a, en effet, cessé d'exhumer des opuscules tardo-antiques ou médiévaux, le plus souvent traduits du grec, et largement inconnus de la critique moderne. Or, pour insignifiantes qu'elles parussent aux yeux des historiens, ces Vies brèves et ces listes de notices n'en offraient pas moins un aperçu inédit sur les sources des auteurs médiévaux en matière de traditions orientales; ce faisant, elles éclairaient aussi la physionomie ancienne de ces mêmes traditions, car beaucoup de traductions latines répertoriées dans ce volume sont plus anciennes que les témoins qui nous sont parvenus de leurs modèles grecs. Enfin, d'un point de vue plus strictement philologique, en tirant de l'oubli ces opuscules latins, FD n'a pas peu contribué à clarifier des traditions textuelles que l'absence de ces chaînons essentiels rendait impénétrables. 
2 Ce volume réunit donc 12 études - éditions, Quellenforschung, répertoires critiques dont une, la dernière, était inédite; les 11 premières avaient été publiées entre 1986 et 2008, mais 3 d'entre elles (II, VI, VII) ont été entièrement refondues et actualisées ; les autres ont plus simplement été enrichies d'addenda (actualisation bibliographique, précisions, corrections, compléments), regroupés en fin de volume. Celui-ci est organisé en 2 parties. La première (I-V) traite des Vies brèves de prophètes et d'apôtres, la seconde (VI-XII) recense et édite des opuscules - ou «listes » - formés de courtes notices, souvent apocryphes, sur les apôtres et les 70 (ou 72) disciples du Christ. Le recueil est utilement complété par 4 index (noms de prophètes, d'apôtres et de disciples, noms d'auteurs et d'œuvres, autres noms propres, index des mss) ; l'avantpropos offre une liste commentée des opuscules étudiés, avec une brève synthèse des résultats obtenus, des questions encore pendantes et des nouvelles découvertes encore escomptées à ce jour.

3 I/ « Deux opuscules latins, relatifs aux personnages de la Bible et antérieurs à Isidore de Séville ». En 1986, FD édita 2 opuscules tardo-antiques, l'un attribué à Épiphane de Salamine, l'autre intitulé De ortu et obitu prophetarum et apostolorum (Doopa), et qui résultaient, au moins partiellement, de traductions du grec; il démontra que ces textes avaient été utilisés par Isidore et que Doopa était également connu du traité pseudoisidorien De ortu et obitu patriarcharum (Doop2).

4 II/ « Nouvelles recherches sur le De ortu et obitu prophetarum et apostolorum ». En 1992, FD réédita l'opuscule Doopa, sur la base d'une tradition manuscrite élargie. La découverte de 3 mss supplémentaires l'amène aujourd'hui à en offrir une troisième édition, avec un commentaire actualisé et révisé à la lumière de ses dernières recherches.

5 III/ "Sur les miracles d'Élie et Élisée (CPL 1155e)». Cette étude, co-publiée avec É. Poirot, était consacrée à un opuscule africain $\mathrm{du}_{\mathrm{IV}}^{\mathrm{e}}$ ou $\mathrm{v}^{\mathrm{e}} \mathrm{s}$., les Virtutes Heliae et Helisaei, dont l'article fournissait une édition et une traduction. Dans ses addenda, FD signale la découverte de 2 nouveaux mss, dépourvus toutefois d'incidence majeure sur le texte édité en 1994.

6 IV/ «Comment travaillait un compilateur de la fin du VIII ${ }^{e}$ siècle $:$ la genèse du De ortu et obitu patriarcharum du Pseudo-Isidore ». L'opuscule Doop2 fut composé au viII ${ }^{\mathrm{e}}$ s. dans un milieu irlandais d'Allemagne du Sud. En 1998, FD identifia, parmi les mss de cet opuscule, le dossier de travail du compilateur.

7 V/ «De uita et obitu prophetarum. Une traduction médiolatine des Vies grecques des prophètes ». Cette étude, parue en 1990, contient l'édition d'un opuscule traduit du grec, vraisemblablement durant le Moyen Âge central, par un compilateur des Balkans. Dans ses addenda, FD estime aujourd'hui que le texte pourrait être d'origine vénitienne.

8 VI/ «Listes d'apôtres et de disciples ». Cet article, d'abord publié en 2005, a été révisé et augmenté pour le présent volume. Il consiste en un tableau actualisé des principales listes grecques: deux listes anonymes tardo-antiques (modèle grec de l'Anonyme de Turner, Index graeco-syrus de Schermann), une liste attribuée à Épiphane de Salamine, et, enfin, une liste compilée à la fin du viII ${ }^{\mathrm{e}}$ s. par un faussaire, qui la plaça sous le nom du martyr Dorothée de Tyr. Une bibliographie sélective et une traduction française des trois premières listes complètent ce panorama.

9 VII/ «Listes latines d'apôtres et de disciples, traduites du grec ». Cette étude constitue le pendant latin de la précédente. Basée sur un article de 1992, entièrement revu pour 
l'occasion, elle consiste en un inventaire critique de 9 listes latines dérivées de modèles grecs. En appendice, FD a reproduit l'Anonyme de Turner, et édité un Index discipulorum qui circulait sous le nom de Jérôme dans les mss d'Italie méridionale : il s'agirait en fait d'une traduction de l'Index graeco-syrus, effectuée entre le vi ${ }^{\mathrm{e}}$ et le viII $\mathrm{e}$. VIII/ "Une liste ancienne d'apôtres et de disciples traduite du grec par Moïse de Bergame ». En 1986, FD découvrit une traduction latine inédite de la liste grecque qui circulait en Orient sous le nom d'Épiphane. Cette traduction avait été réalisée, sans doute à Constantinople, par l'Italien Moïse de Bergame (XII ${ }^{\mathrm{e}} \mathrm{s}$.).

11 IX/ «Une liste latine de disciples et d'apôtres traduite sur la recension grecque du Pseudo-Dorothée ». Du Pseudo-Dorothée, on connaissait déjà une traduction latine, effectuée en Italie (BHL 654kj) ; en 1990, FD en découvrit une autre, indépendante de la première ; également italienne, elle aurait été réalisée au XII ${ }^{\mathrm{e}} \mathrm{s}$., en Vénétie.

12 X/ «Une liste latine d'apôtres et de disciples compilée en Italie du Nord ». En 1998, FD édita une nouvelle liste d'apôtres et de disciples, transmise sous le nom de Jérôme, et compilée entre 850 et 1150 environ, à partir de différentes sources latines. Ce montage n'aurait pas présenté grand intérêt s'il n'avait pas constitué le premier jalon d'un culte de Barnabé à Milan, et donc des prétentions apostoliques de cette cité à l'époque médiévale.

$13 \mathrm{XI} /$ "Trois témoins méconnus de l'Index discipulorum du Pseudo-Dorothée ( $\mathrm{XIV}^{\mathrm{e}}-\mathrm{XVI}^{\mathrm{e}}$ s.) ». Cet article, paru en 2008, était consacré à la tradition indirecte d'une traduction perdue du Pseudo-Dorothée. Outre les deux opuscules mentionnés plus haut (IX), une troisième traduction, encore introuvable, fut en effet utilisée par les légendiers abrégés des Vénitiens Pierre Calo et Pierre Natal ( $\mathrm{XIV}^{\mathrm{e}} \mathrm{s}$.). Lors du concile de Trente, cette traduction perdue inspira également un programme iconographique à Reichenau.

XII/ « Nouvelle liste latine d'apôtres et de disciples originaire d'Italie du Nord ». Dans cette étude inédite, FD étudie et publie, d'après son unique témoin (London, BL, Add. 14092), une liste du XIV $\mathrm{s}$., compilée en Vénétie d'après une marqueterie de sources latines. Le lecteur trouvera également, en appendice, les éditions de travail de deux listes en rapport avec cette nouvelle liste italienne : la liste brève de Bernard Gui et celle de Paulin de Venise. Dans ses addenda, FD a également ajouté une brève liste de disciples évangélisateurs des Gaules, transmise par un ms. de Saint-Victor de Paris (Paris, Mazarine, 1730, XIV s.).

Avec ce large panorama, FD entendait planter les jalons nécessaires à la reprise d'un projet collectif déjà ancien, celui de rééditer, dans toutes les langues de la tradition chrétienne, le vaste dossier des vies brèves de prophètes et d'apôtres. Force est de constater que son apport, côté latin, demeurera déterminant. Ce volume, en effet, ne se contente pas de rassembler commodément des études anciennes; l'apport inédit (addenda, éditions nouvelles, refonte complète de certains articles) y est plus que significatif, et la cohérence de l'ensemble, remarquable. Plus qu'un recueil d'articles, ce volume des "Subsidia hagiographica » sera donc un guide de référence et un outil de consultation indispensable sur le dossier des Vies brèves de prophètes et d'apôtres. Mais l'intérêt de cet ouvrage dépasse ce seul domaine érudit. Entre 1986 et aujourd'hui, FD n'a en effet cessé de paver son enquête de nouvelles découvertes. Aussi son livre donne-t-il à voir tout le cheminement qu'il a suivi, un chemin non pas solitaire, mais enrichi des progrès d'autres chercheurs, dont les découvertes ont nourri et stimulé, au fil des années, sa propre réflexion. Cela rend son volume exemplaire, non seulement 
par sa qualité scientifique, la précision et la clarté des analyses qui y sont conduites, l'acribie des éditions qui y sont proposées, mais aussi parce qu'on y trouvera le reflet sobre et formateur de tout un parcours de recherche, marqué au sceau de la patience et de l'exigence scientifique.

\section{AUTEURS}

\section{CÉCILE LANÉRY}

Institut de Recherches et d'Histoire des Textes, Paris. 